\title{
It is not the time for science fiction
}

\author{
Government officials who disseminate unsupported claims about the novel coronavirus undermine public trust in \\ science and in the public-health efforts that are essential to bringing the COVID-19 pandemic under control.
}

T ransparency has not been a hallmark of the Trump administration's response to the COVID-19 pandemic in the USA. Public criticism has mounted about the slowness of the US government in recognizing the threat of the coronavirus, its disregard for evidence-based recommendations by the World Health Organization and the unclear source of research backing up many of US President Trump's recommendation to the public. Now that it is increasingly clear that SARS-CoV-2 - the coronavirus that causes COVID-19 - will be around for the long run and that the economy might not recover from the lockdown effect anytime soon, in the USA, deflection is giving space to distortion of scientific evidence to fulfill a political agenda, at the risk of undermining public trust in health experts and in science itself.

In question, among other issues, are the origin of SARS-CoV-2 and claims that it was created in a lab in China, as well as whether the Chinese government concealed critical information in the early days of the Wuhan outbreak. Understanding how pandemics begin is important. This knowledge can help countries to prepare for future outbreaks and hopefully to prevent them from reaching pandemic proportions. It is also a fair question to be asked by anyone whose life has been upended by the virus: could all of this have been avoided? There is no easy answer. Scientists have been working since the early days of the pandemic to decode the genome of SARS-CoV-2, to compare it with those of related viruses found in wild animals and to try to map possible evolutionary routes and jumps between species. The best scientific evidence available thus far supports the idea that SARS-CoV-2 originated in nature, probably in bats, and that it may have made its way to humans through another intermediary animal. Some researchers have tried to identify 'marks' of human manipulation in the genome of the new coronavirus as evidence that the virus was engineered in a lab. They found none. Much more research is needed to truly understand the biology of the new coronavirus, and it is important to convey to the general public that scientific knowledge is always evolving as more data become available. It is worrisome, however, that in stark contrast to the best evidence available and the opinion of most experts, high-ranking members of the US government continue to propel a narrative in which the virus was manmade in China.

The US government is hardly alone in its strategy for deflecting attention from its poor performance in the current pandemic. Disseminating falsehoods and fear exploitation have become a common approach for distracting the general population from governance deficiencies in the response to COVID-19. In response to US criticism of China's lack of transparency about what is known about the early spread of the outbreak, Chinese officials have pushed the idea the virus was introduced in Wuhan by the US Army in October 2019. Claims that the virus was created as a bioweapon have surfaced in Venezuela and Iran and are taking over the official narrative in Russia. In Brazil, which at the time of this writing has the second-worst outbreak in the world, the Bolsonaro administration - which is openly anti-science - has followed the Trump playbook in downplaying the severity of the threat, promoting unproven treatments for COVID-19 and disregarding scientific evidence that supports the critical role of social isolation and lockdown measures in slowing the spread of the virus. While scientific skepticism is not new, the overwhelming volume of misinformation and the amount of official government promotion of fringe science seems unprecedented, facilitated by the interconnectivity of the contemporary world.

Co-opting science to support a politically motivated narrative of the pandemic has consequences for everyone. In April 2020, the National Institute of Allergy and Infectious Diseases, part of the US National Institutes of Health, abruptly ceased funding the research of scientists at EcoHealth Alliance, who were collaborating with Chinese scientists in Wuhan (among others) on research to understand how coronaviruses can move from bats to humans. This decision by the US National
Institutes of Health, which was scientifically unjustified and was widely criticized by the research community, set a dangerous precedent for political interference in the scientific enterprise, beyond hindering the progress of research in an area that is critical to informing public-health preparedness for future pandemics. The feud between the USA and China also undermines the goal of establishing international collaborations that will be needed in order to understand the evolution of the COVID-19 pandemic and find solutions for the challenges ahead - a critical commitment firmed at the World Health Assembly in May. The most important consequence of a government leader's using the pandemic narrative for political gain, however, is the progressive loss of public trust in science and in the guidance of experts.

Getting back on the right track will not be easy and will require a multipronged approach. Science still has the momentous advantage of being recognized as a critical element in the solution of the COVID-19 crisis. The research community can and should influence public opinion. To do so, researchers must remain engaged with the public discussion on critical aspects of the pandemic, informing the population, holding the public officials accountable for their policy decisions, debunking conspiracy theories, pursuing international collaborations and reacting to further attempts to distort the scientific record. Sustainable control of the coronavirus, in the USA and elsewhere, will be achieved only through public-health interventions - be those quarantines, social distancing, contact tracing, treatment or vaccination. In the long run, in free societies, in which people have the freedom to choose, the success of these interventions is dependent on public trust in scientific experts and health officials. It is crucial that government leaders set politics aside and allow science to guide policy decisions and support international collaborations to bring a swift end to this pandemic. 